\section{Primary malignant melanoma of the oesophagus: case report and review of surgical pathology}

\author{
G J Stranks, J T Mathai, D C Rowe-Jones
}

\begin{abstract}
Primary malignant melanoma of the oesophagus is both a rare presentation for melanoma and a cause of oesophageal neoplasm. It accounts for less than $0 \cdot 1 \%$ of all primary oesophageal neoplasms. It was first recognised as a primary tumour by de la Pava $e t$ al who showed the presence of melanocytes within oesophageal mucosa. This case report includes a review of the surgical pathology. Even though it is a rare lesion it must be considered as a cause of polypoidal oesophageal lesions as both its diagnosis and treatment can present problems. It is usually fatal within one year.
\end{abstract}

Primary malignant melanoma of the oesophagus is both a rare presentation of melanoma and a rare cause of polypoidal oesophageal tumour. Its pathological diagnosis can present a problem and despite treatment with radical resection the condition is normally fatal within one year.

\section{Case report}

In December 1988, a 64 year old white woman presented to her general practitioner with a 10 month history of weight loss, retrosternal pain, and mild difficulty in swallowing. A barium swallow was arranged (Fig 1) and this showed a rounded filling defect at the junction of the middle and lower third of the oesophagus. At the time this was thought to represent a lieomyoma rather than a neoplastic lesion.

Gastroscopy confirmed the presence of a polypoidal lesion at $25-30 \mathrm{~cm}$, which bled on surface contact. There was no major oesophageal obstruction and the gastroscope passed easily beyond the lesion. Biopsy specimens suggested a diagnosis of malignant melanoma and raised the question as to whether this was a primary or a secondary lesion.

Repeat clinical examination of the patient showed that there were no suspicious pigmented lesions of the skin or eyes and was otherwise unremarkable.

A computed tomogram of the chest and abdomen confirmed a mass in the middle third of the oesophagus that was compressing and distorting the lumen. No extension into the surrounding tissues was seen and there was no involvement of the mediastinal or para-aortic nodes (Fig 2).

An Ivor Lewis oesophagectomy was per- formed with macroscopically complete excision of the lesion. The patient made a good recovery and was discharged home on day 16 after surgery.

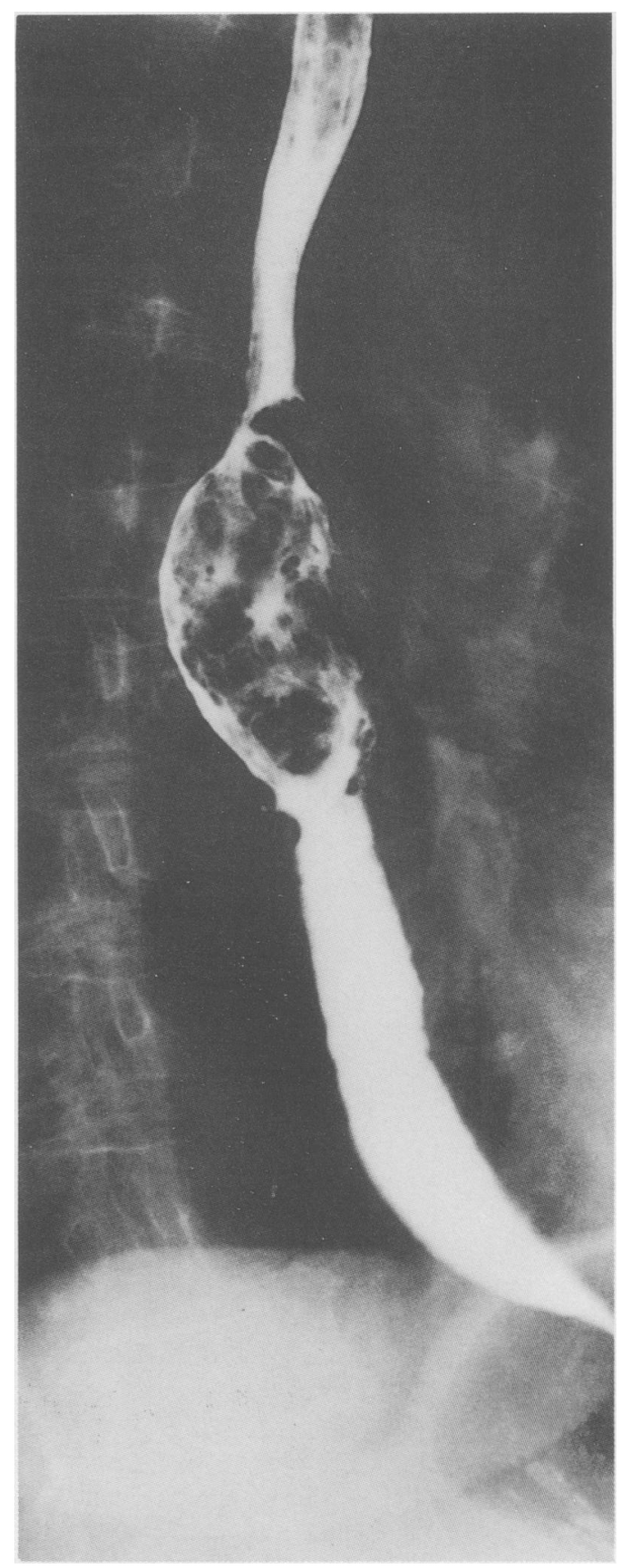

Figure 1: Barium swallow showing polypoidal tumour as a filling defect.
Surgery Poole Geneal Hospital, Poole, Dorset J T Mathai DC Rowe-Jones Mr G J Stranks, Rivendell, Chase Grove, Waltham Chase

Accepted for publicatio 3 August 1990 


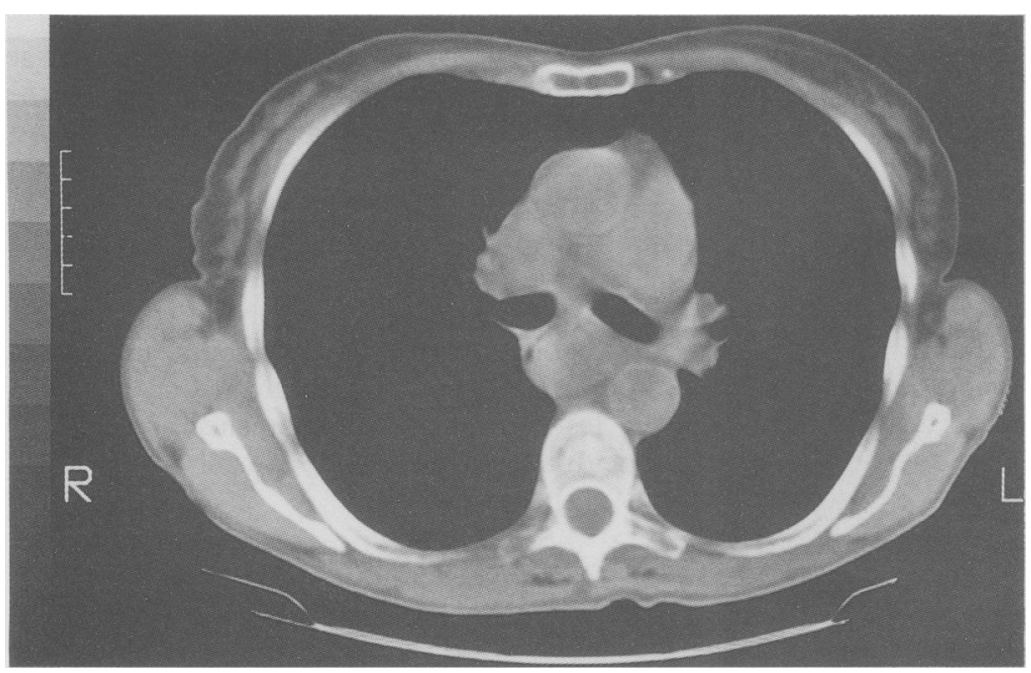

Figure 2: Computed tomogram confirming tumour. No lymphatic involvement seen.

Macroscopic pathological assessment showed a $10 \mathrm{~cm}$ length of oesophagus containing two nodules. The larger was pigmented black, the smaller pale, and both were ulcerated (Fig 3). Neither seemed to have penetrated the oesophageal wall.

Microscopic examination showed that the nodules consisted of malignant melanoma that had not penetrated through the mucosal wall. All lymph nodes were clear. Junctional activity was noted in the adjacent oesophagus. This is a feature of a primary lesion and it was therefore reported as primary malignant melanoma of the oesophagus (Fig 4).

The lady remained well for a period of five months but then presented with multiple subcutaneous nodules on her chest wall and abdomen. A biopsy specimen of one of the nodules showed metastatic malignant melanoma. A gastroscopy was then performed, but no recurrence at the primary site was found.

\section{Review of malignant melanoma of the oesophagus}

HISTORY

The first description of primary malignant melanoma of the oesophagus was by Baur in

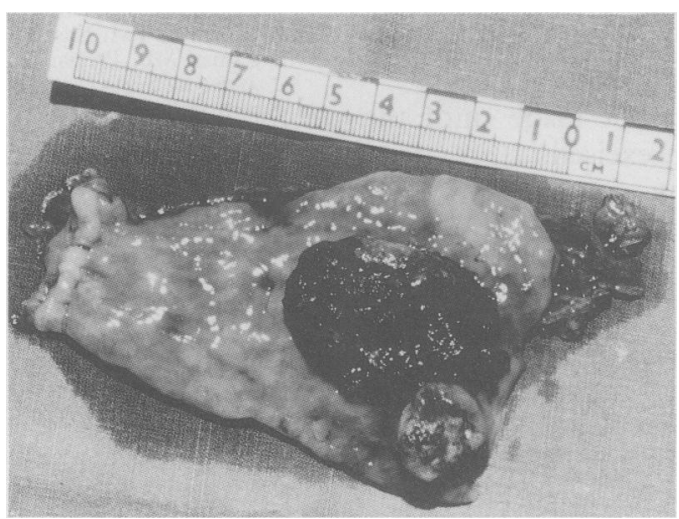

Figure 3: Primary melanoma of the oesophagus after resection.
1906. The first case, however, was reported in 1952 by Garfinkle and Cahan,' although its existence was doubted since melanocytes had not been identified within the oesophageal wall. When de la Pava et $a l^{2}$ showed typical melanoblasts in four out of 100 oesophagi from necropsy material in 1962 the diagnosis was accepted.

\section{EPIDEMIOLOGY}

This is a rare tumour accounting for only $0 \cdot 1-$ $0 \cdot 2 \%$ of all oesophageal tumours, ${ }^{34}$ and there have been only 100 or so cases reported to date. When Turnbull et $a l^{3}$ reviewed 1918 cases of oesophageal tumours between 1926 and 1968, they found only two cases of malignant melanoma. Even secondary spread of malignant melanoma, which is common within the gastrointestinal tract, is uncommon in the oesophagus, only $4 \%$ were found in a large study of disseminated melanoma.

These tumours seem to occur more often in men than women, (approximately $2: 1^{13}$ ) and generally occur from the 50th decade onwards, although one case has been reported in a child. ${ }^{56}$

The origin of the tumour remains largely speculative. It has been suggested that it arises within ectopic melanocyte precursors which migrate down the upper two thirds of the oesophagus during development. It has also been suggested that melanosis of the oesophagus could be a predisposing factor since it is present in about $25 \%$ of affected cases. ${ }^{56}$

\section{PATHOLOGY}

Macroscopically, primary tumours tend to be large, stenosing, polypoidal, and are often focally ulcerated. This is the case in about $25 \%$ of patients. They present either as a single lesion or one with smaller satellites. They can range in colour from white to black-brown. The pigmentation seen can be melanin but equally it can be the result of secondary haemorrhage within the tumour. ${ }^{14}$

When the tumour is the result of secondary spread from a cutaneous primary it can mimic this picture. More often however, the lesions are smaller, more nodular, and more widespread.

The microscopic criteria for deciding whether the lesion is a primary tumour were set out by Raven and Dawson in 1964," based on the proposals of Allen and Spitz. They stated that the tumour should have the structure of a melanoma, contain melanin granules, and most importantly should arise from an area of junctional change within squamous mucosa and that the adjacent epithelium should also show nests of atypical melanocytes with pleomorphic nuclei that is junctional changes. They suggested that these findings should be present before the lesion may be labelled a primary melanoma. ${ }^{12}{ }^{1+}$ Some argue, however, that a rapidly growing tumour may sometimes obliterate the adjacent junctional changes.

Growth and spread occur mainly within the submucosa, and despite the size of an often large tumour they can be covered with intact squamous mucosa. It is for this reason that tumour extent is often underestimated on gross examination. 


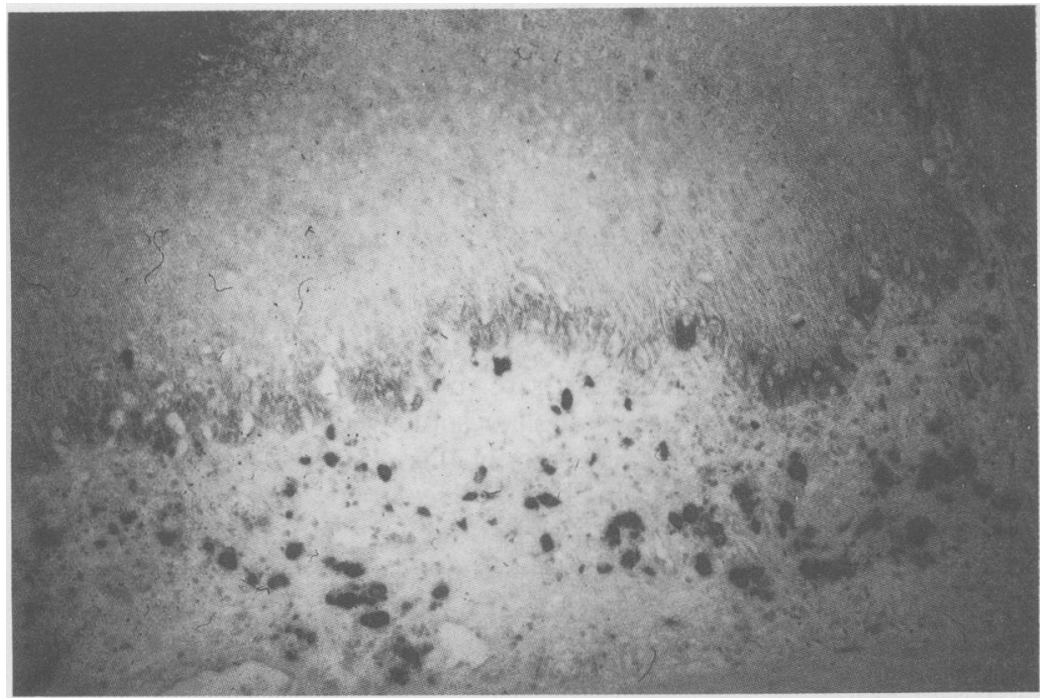

Figure 4: High power microscopy of oesophageal wall adjacent to the tumour showing junctional activity with nests of atypical melanocytes. (Original magnification $\times 128$.)

In terms of secondary spread they behave very much like other melanomas with blood borne metastasis and lymph node involvement.

\section{CLINICAL FEATURES}

The commonest presenting symptoms are dysphagia and weight loss. Other symptoms include discomfort on swallowing or retrosternal or back pain, or both. There is usually a relatively short history of only a few months' duration. ${ }^{8}$

\section{DIAGNOSIS}

As always, a detailed history and clinical examination is important in distinguishing between primary and secondary disease. It is important to exclude a cutaneous primary tumour.

A barium swallow will usually show a large, exophytic, lobulated, ulcerating polypoidal mass in the mid- to lower oesophagus. ${ }^{8}$

Gastroscopy can be both useful and misleading as biopsy specimens can be misinterpreted as poorly differentiated squamous cell carcinoma. This is because of the submucosal nature of the tumour and the fact that the melanoma cells may contain little or no melanin pigment. ${ }^{14}$ Immunohistochemistry and electron microscopy have been described as useful in distinguishing between primary and secondary disease if melanoma is recognised at biospy. ${ }^{59}$

The diagnosis is often not made until the tumour has been surgically excised.

Computed tomography is an extremely accurate way of assessing the oesophageal lesion with regard to size, shape, degree of local spread, and lymph node involvement.

\section{DIFFERENTIAL DIAGNOSIS}

The differential diagnosis is from any large polypoidal lesion occurring within the oesophagus whether benign or malignant. Most are benign and include leiomyomas and lipomas that can ulcerate, but fibromas, neurofibromas, and fibrovascular polyps are also possible. The malignant group include squamous cell carcinoma, leiomyosarcoma, rhabdomyosarcomas, epidermoid and small cell carcinoma, and adenocarcinoma arising from a Barrett's oesophagus. The lesion must be distinguished from metastatic tumour. ${ }^{5812}$

\section{TREATMENT AND PROGNOSIS}

The treatment of any primary malignant melanoma is wide surgical resection. This is the case for all primary malignant melanomas of the oesophagus, and is the treatment of choice where possible. An oesophagectomy is performed with restoration of gut continuity as much to relieve symptoms as to establish a cure.

Some authors advocate postoperative radiotherapy in selected patients, ${ }^{10}{ }^{13}$ while others have tried repeated resection ${ }^{9}$ in an attempt to reduce recurrence locally. Overall, however, neither chemotherapy nor radiotherapy have affected mortality appreciably. ${ }^{7}$ Immunotherapy is a theoretical possibility and has been reported as improving prognosis but has not been fully evaluated. ${ }^{13}$

The tumour is highly malignant and has a poor prognosis with an average survival of seven to 12 months. Of the cases reported, $30-40 \%$ have metastases at presentation and five year survival is quoted at $4 \cdot 2 \% .{ }^{14}$ Published results in 1956 compared with those published recently show little difference. ${ }^{512}$ Early death with widespread metastases is the usual final outcome.

1 Garfinkle JM, Cahan WG. Primary melanocarcinoma of the esophagus. First histologically proven case. Cancer 1952; 5: 921-6.

2 de la Pava S, Nigogosyan G, Pickren JW, Cabrera A. Melanosis of the esophagus. Cancer 1963: 16: 48-50.

3 Turnbull AD, Rosen PP, Goodner JT, Beattie EJ. Primary malignant tumours of the esophagus other than typical epidermoid carcinoma. Ann Thorac Surg 1973; 15: 463-73.

4 Chalkiadakis G et al. Primary malignant melanoma of the esophagus. Ann Thorac Surg 1985; 39: 472.

5 DiCostanzo DP, Urmacher C. Primary malignant melanoma of the esophagus. Am $\mathcal{F}$ Surg Path 1987; 11(1): 46-52.

6 Ludwig ME, Shaw R, Suto-Nagy G de. Primary malignant melanoma of the esophagus. Cancer 1981; 48: 2528-34.

7 Das Gupta TK, Brasfield R. Metastatic melanoma: A clinical pathologic study. Cancer 1969; 17: 1323-39.

8 Jensen D, Stark P, Gardiner G, Hyde M. Primary malignant melanoma of the esophagus. ROFO 1988; 149(3): 330-1.

9 Farsad H, Reza G. Primary malignant melanoma of the esophagus. South Med F 1980; 73(11): 1535-7.

10 McCormack PM, Nasciminto AG, Bains MS, Knapper WH, Zaman MB. Primary melanocarcinoma of the esophagus. Memorial Sloan Kettering Cancer Bulletin 1979; 9: 162-4

11 Raven RW, Dawson A. Malignant melanoma of the oesophagus. Br F Surg 1964; 51: 551-5.

12 Sostman HD, Koehana MF, Lee CH, Curtis AM, Farman J. Primary oesophageal melanocarcinoma. $\mathrm{Br} \mathcal{F}$ Radiol 1980 ; 53(630): 589-92

13 Roesch W, Roher HG. Primary malignant melanoma of the oesophagus. Endoscopy 1984; 16: 186-8.

14 Simpson NS et al. Primary malignant melanoma of the oesophagus. F Clin Path 1990; 43: 82-4. 